\title{
Characterization of a conveyance device based on a digital actuators array and a structured plate
}

\author{
Jing XU, Laurent PETIT, Erwan DUPONT, Christine PRELLE \\ Sorbonne universités, Université de technologie de Compiègne, CNRS, UMR 7337 Roberval, \\ Centre de recherche Royallieu - CS 60319 - 60203 Compiègne cedex, France \\ jing.xu-j@utc.fr, laurent.petit@utc.fr, erwan.dupont@utc.fr, christine.prelle@utc.fr
}

\begin{abstract}
This paper presents a conveyance device based on a digital actuators array and a structured plate. The actuators array is composed of four electromagnetic digital actuators, arranged in a $2 \times 2$ matrix. The principle of the actuators array and of the conveyance device are firstly presented. The control strategy of the array to generate the conveyance task is then described. A prototype of the device is presented and experimentally tested. Conveyance of the structured plate has been experimental realized in different conditions and compared with theoretical results.
\end{abstract}

Keywords-Digital actuators array; Electromagnetic digital actuator; Conveyance device; Structured plate

\section{INTRODUCTION}

Mechatronic systems trend to become more complex due to the need of increasing functionalities. To achieve this objective and to ensure high reliability levels, actuators have to be integrated together with sensors. Moreover, the size of these mechatronics systems decrases more and more due to the high integration constraints.

The actuators can be classified into two categories according to their functioning: the analogical and digital actuators. Analogical actuators can reach any position located between the extreme positions of their stroke. These actuators are generally controlled in closed loop with sensory components to ensure high performances. However, the added sensory components make their integration more complex, or not always possible, especially in the case of compact or highly integrated mechatronics systems.

An alternative to these actuators can be found with the use of digital actuators. Digital actuators are composed of a mobile part which can switch between several precise and repeatable discrete positions [1]. All intermediate positions, located between these discrete positions, correspond only to transient states and cannot be held in normal functioning. The control of these actuators is very simple and can be realized in open loop without feedback sensors [2]. Thus, the control of digital actuators is based on energy pulses only needed to switch the mobile part between the discrete positions. The control of this kind of actuators can then be realized using digital outputs of a data acquisition board (instead of analog outputs) that represents a lower cost for the control. For all these reasons, the integration of digital actuator in mechatronical system becomes much easier. Besides, the energy consumption is minimized because energy is only consumed during the switching and no external energy is consumed to hold the mobile part in discrete position. However, the main drawback of digital actuators is their discrete strokes defined during the manufacturing step. Using several digital actuators, variable strokes can nevertheless be obtained. Moreover, an important constraint of digital actuator is the manufacturing quality which should be very important because manufacturing errors cannot be compensated using the control as for analogical actuators.

In literature, the digital principle has been widely studied and used. The switching function of these actuators can be obtained by several physical principles, such as electrothermal [3], electrostratic [4], electromagnetic [5], [6] and piezoelectric effects [7]. Digital actuators are widely used in different domains. The applications can be divided into two groups according to the number of actuators: single actuator and actuators array. Single actuators are typically used for switches for fluidic [8], optical [9], [10] and electrical [11], [12] applications. Digital actuators can also be used as array to perform more complex tasks by combing several elementary actions. The common applications of actuators arrays are optical switch arrays [13], tactile displays [14], digital robots [15] and displacement tables [16], [17].

In this paper, a Digital Actuators Array (DAA) composed of four Elementary Digital Actuators (EDA) arranged in a $2 \times$ 2 matrix is presented. The electromagnetic principle has been chosen for the EDA because it is well adapted to digital actuation. The holding function can indeed be easily obtained using Permanent Magnets (PMs). An application of this DAA as a conveyance device is proposed in the paper. In Section 2, the principles of the EDA, with four discrete positions, of the DAA and of the conveyance device are introduced. In section 3 , a prototype of the conveyance device is described and some experimental tests in different conditions are presented. Experimental results are then compared with theoretical results.

\section{PRINCIPLE OF THE CONVEYANCE DEVICE}

In this section, the principles of the EDA and of the DAA are firstly presented. Then, the principle of the conveyance device and the associated controlling sequence of the DAA are described.

\section{A. Principle of the $D A A$}

A schematic layout of the EDA is presented in Figure 1. This actuator is composed of a NdFeB Mobile Permanent 
Magnet (MPM) placed in a square cavity. The MPM can reach four discrete positions located at each corner of the cavity. Two orthogonal sets of five serially connected electrical wires are used to switch between the discrete positions. When a current passes through a set of wires, a Lorentz force is generated on it. Because the set of wires is fixed, the resulting force is exerted on the MPM. The two sets of wires have been printed on a double-side Printed Circuit Board (PCB). The set of wires to switch along $\mathrm{y}$-axis is placed at a shorter distance to the bottom side of the MPM ( $d_{2}$ in Fig. 1 (b)) than the set to switch along $\mathrm{x}$-axis $\left(\mathrm{d}_{3}\right)$ because it is printed on the top side of the PCB. The electromagnetic force generated is then higher along y $(8.85$ $\mathrm{mN}$ with $1 \mathrm{~A})$ than along $\mathrm{x}$-axis $(8.36 \mathrm{mN}$ with $1 \mathrm{~A})$. To obtain a magnetic holding of the MPM in each discrete positions, four Fixed Permanent Magnets (FPMs) are placed around the cavity. When the MPM is located in a discrete position, the magnetic holding force exerted on it is $16.85 \mathrm{mN}$. The MPM and FPMs dimensions (magnetization) are $10 \times 10 \times 2 \mathrm{~mm}^{3}$ $(1.35 \mathrm{~T})$ and $2 \times 2 \times 2 \mathrm{~mm}^{3}(1.45 \mathrm{~T})$, respectively. The MPM stroke is $4 \mathrm{~mm}$ and the distances $\mathrm{d}_{1}, \mathrm{~d}_{2}, \mathrm{~d}_{3}$ and $\mathrm{d}_{4}$ are: $3,5 \mathrm{~mm}$, $222 \mu \mathrm{m}, 458 \mu \mathrm{m}$ and $1 \mathrm{~mm}$, respectively. The calculation of the magnetic and electromagnetic forces has been realized using the RADIA software. More details on the design of the EDA are given in [18].

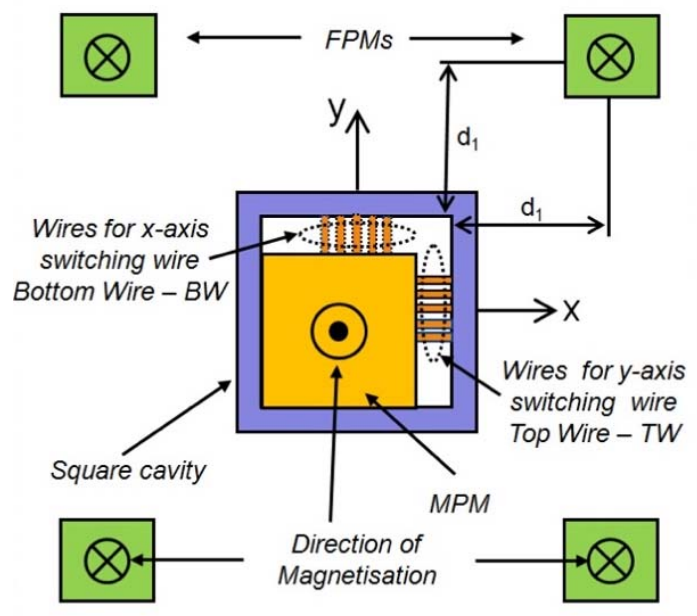

(a)

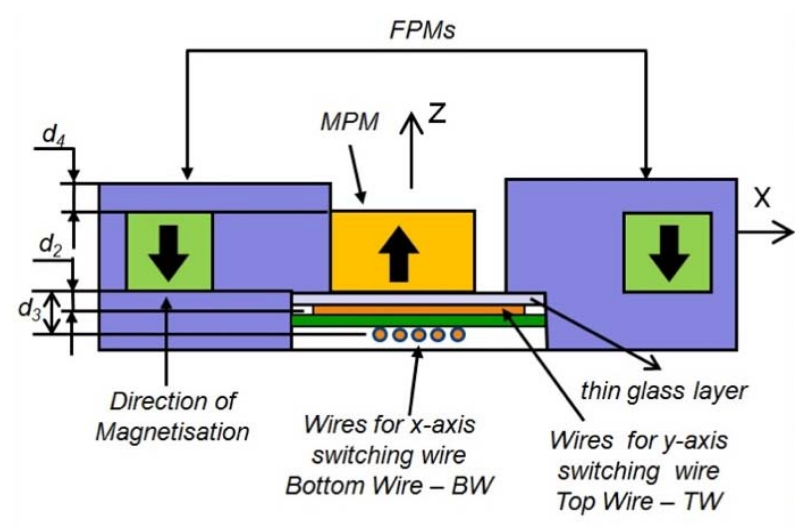

(b)

Fig. 1. Schematic layout of the EDA - a Top view - b Side view
A schematic layout of the DAA is presented in Figure 2. It is composed of four EDAs placed one beside the others and Added Fixed Permanent Magnets (AFPMs) have been added on the boundaries of the EDAs in order to balance the magnetic interactions between the four MPMs with the objective to obtain an homogeneous behavior of each EDA. The distances $\mathrm{d}_{0}$ and $\mathrm{d}_{\mathrm{f}}$ are $15 \mathrm{~mm}$ and $5.2 \mathrm{~mm}$ respectively. More details on the design of the DAA are also given in [18].

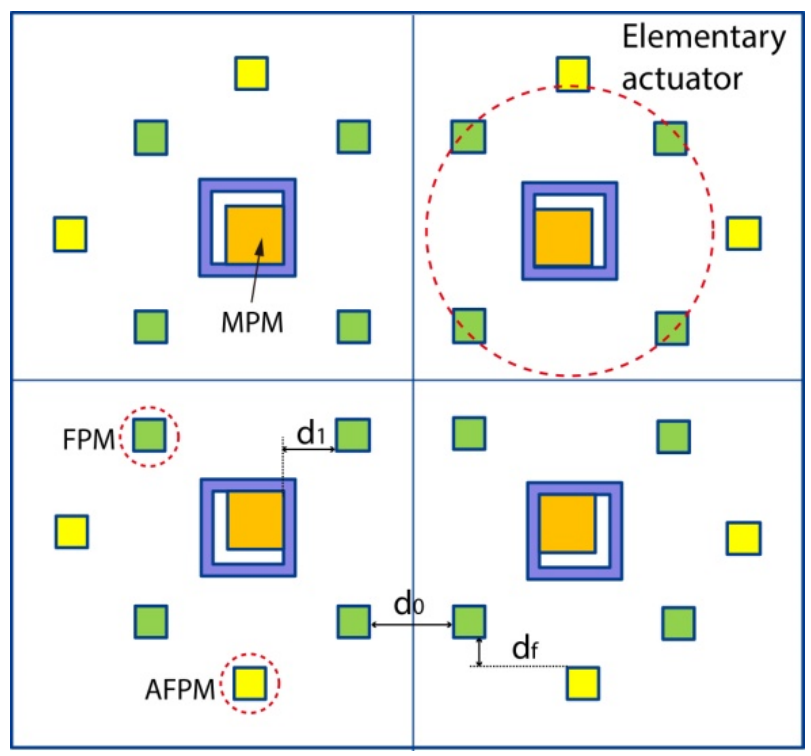

Fig. 2. Schematic layout of the DAA

\section{B. Principle of the conveyance device}

The conveyance device is based on the presented DAA and on a structured plate placed above (Fig. 3). The conveyance principle is based on the rack gear principle. The DAA is used to move the structured plate. For that, each MPM is equipped with a stud on its top side and these studs are used to push the studs on the plate, then to move it. The geometry of the conveyance device (studs dimensions, distance between EDA...) has been selected in order to be able to realize 2D displacements.

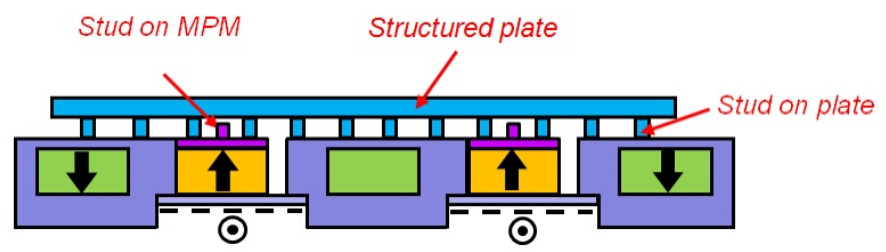

Fig. 3. Schematic layout of the conveyance device with the structured plate

To obtain the conveyance of the structured plate, a dedicated controlling sequence of the four EDAs is used. This sequence is shown in Figure 4. To simplify the understanding, only the studs of the plate and on the MPMs are represented. Initially (Fig. 4(a)), the MPMs are placed in the position placed near to the DAA center. At the first step, the MPMs 3 and 4 are switched along $+y$ and $-y$, respectively (Fig. 4(b)). With this displacement, the studs on the MPMs are engaged between the studs of the structured plate. At the next step (Fig. 4(c)), the MPMs 3 and 4 are switched along $\mathrm{x}$ direction to push the 


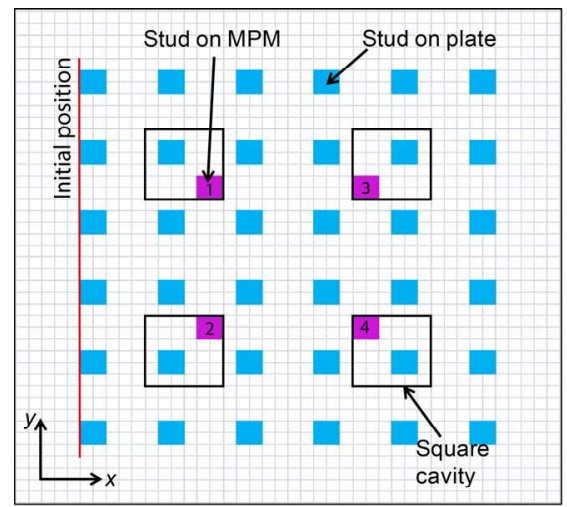

(a)

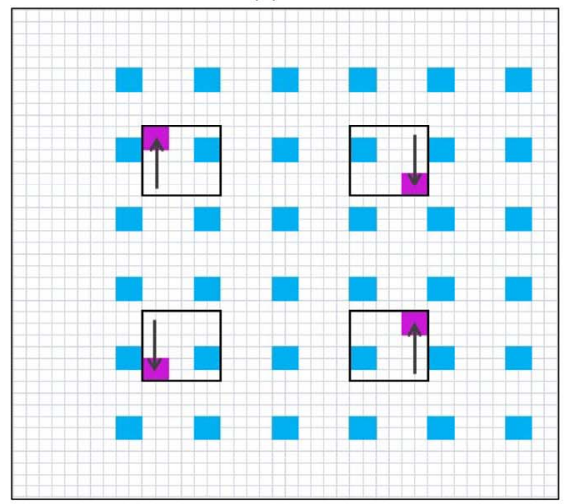

(d)

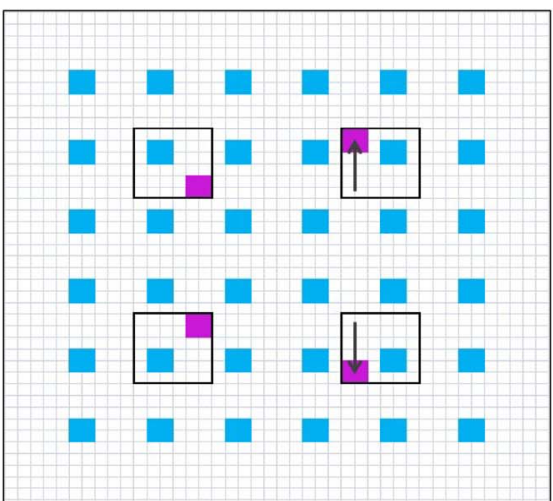

(b)

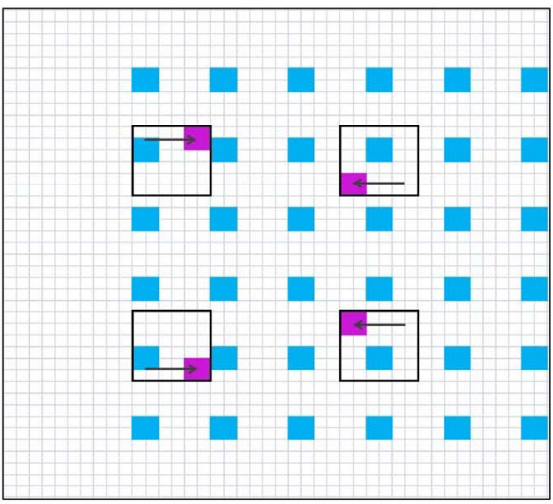

(e)

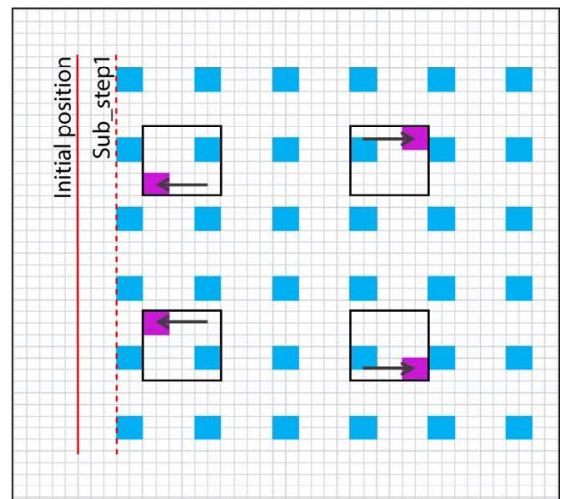

(c)

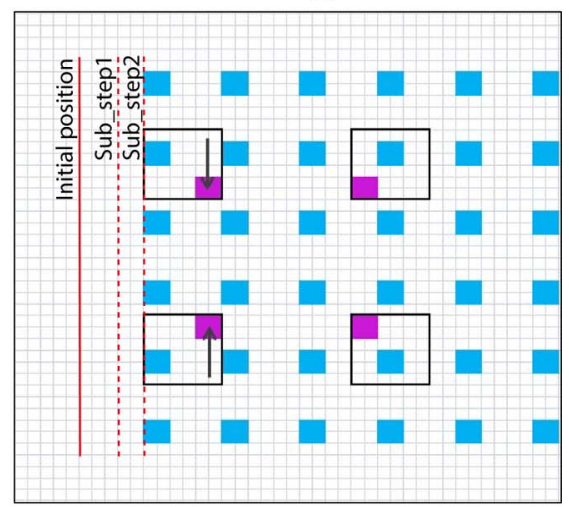

(f)

Fig. 4. Controlling sequence for structured plate displacement

structured plate in this direction. In the same time, the MPMs 1 and 2 are switched along $-\mathrm{x}$ direction in order to place the studs on these MPMs between two studs on the plate (along Xaxis). At the third step (Fig. 4 (d)), the MPMs 3 and 4 are disengaged by switching in the $-y$ direction and in the $+y$ direction, respectively. In the same time, the MPMs 1 and 2 are switched in the $+y$ and $-y$ directions respectively, so that the studs on MPM 1 and 2 are engaged between the structured plate studs. At the fourth step (Fig. 4(e)), the MPMs 1 and 2 are switched along $\mathrm{x}$ direction to push the plate and the MPMs 3 and 4 along $+x$ direction. At the fifth step (Fig. 4(f)), the MPMs 1 and 2 are switched along $y$ axis so that they return to their initial position. Then, a new displacement sequence can be realized. With one controlling sequence, two sub-steps plate displacements are obtained at the second (Fig. 4 (c)) and fourth (Fig. 4 (e)) steps.

\section{EXPERIMENTAL SETUP AND RESULTS}

\section{A. Experimental setup}

Based on a design presented in the previous section, a prototype of the conveyance device has been manufactured using rapid prototyping techniques. The mechanical structure of the DAA (including the square cavities) has been obtained by laser cutting. The structured plate and the studs on the MPMs have been manufactured by 3D printing technique. To control the DAA, four Voltage-to-current converters (V-A converters) are used (2 V-A converters with [-7A ; +7A] output current and 2 with $[-10 \mathrm{~A} ;+10 \mathrm{~A}]$ output current). Each V-A converter is controlled thanks to an analog voltage $[-10 \mathrm{~V}$;
$+10 \mathrm{~V}]$ generated by a data acquisition board (National Instrument (NI) 6289) installed in a computer. Analog control signals have then been used instead of digital control in order to enable the possibility to generate several driving current values during experimentation.
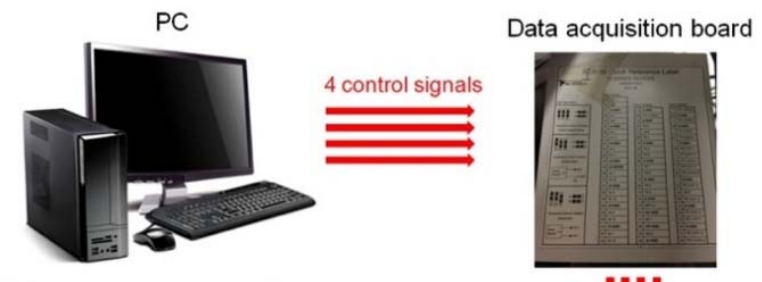

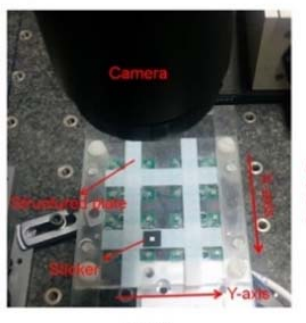

DAA

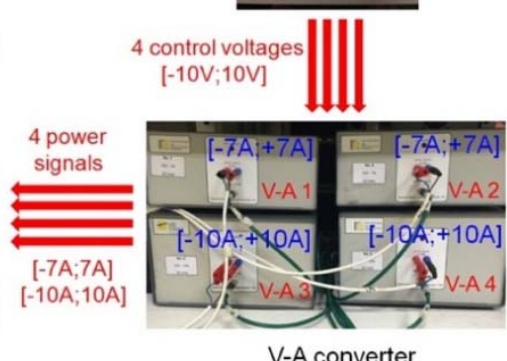

V-A converter
Fig. 5. Experimental setup

A camera placed above the DAA is used to measure the plate displacement. Using the camera, pictures of the plate position are taken during experimentation. Thanks to a calibration of the camera, the plate position can then be obtained. In this case, the limit of resolution of the measure 
(pixel dimension) is $38.5 \mu \mathrm{m}$. The experimental setup is illustrated in Figure 5. On the top side of the structured plate, a sticker, with a white square on a black background, has been pasted. Based on the pictures and using an image processing program developped with MATLAB software, the white square position has been determined for each image.

\section{B. Characterization of the $D A A$}

The proposed conveyance application based on the DAA requires that the behavior of each EDA is identical to the other ones. However, the manufacturing uncertainties of the mechanical parts and of the PMs introduce variation on the EDA behaviors. In order to characterize this variation, an experimental test has been realized without considering the structured plate. In this test, the minimum driving current needed to switch each MPM has been experimentally determined. The results are presented in Table I.

TABLE I. MINIMUM DRIVING CURRENTS NEEDED TO SWITCH THE MPMS ALONG X AND Y AXES

\begin{tabular}{|c|c|}
\hline MPM 1 & MPM 3 \\
\hline $\mathrm{I}_{1 \mathrm{x}}=2.10 \mathrm{~A}$ & $\mathrm{I}_{3 \mathrm{x}}=2.15 \mathrm{~A}$ \\
$\mathrm{I}_{1 \mathrm{y}}=1.90 \mathrm{~A}$ & $\mathrm{I}_{3 \mathrm{y}}=2.05 \mathrm{~A}$ \\
\hline MPM 2 & MPM 4 \\
\hline $\mathrm{I}_{2 \mathrm{x}}=2.00 \mathrm{~A}$ & $\mathrm{I}_{4 \mathrm{x}}=2.10 \mathrm{~A}$ \\
$\mathrm{I}_{2 \mathrm{y}}=1.80 \mathrm{~A}$ & $\mathrm{I}_{4 \mathrm{y}}=1.95 \mathrm{~A}$ \\
\hline
\end{tabular}

For the two displacement axes, the variation of the minimum driving current between the four EDAs is $0.15 \mathrm{~A}$. The average minimum driving currents are $2.09 \mathrm{~A}$ and $1.93 \mathrm{~A}$ for $\mathrm{x}$ and $\mathrm{y}$ axis, respectively. Using an analytical model for magnetic and electromagnetic force calcultation [17], the theoretical minimum driving currents needed to switch have been determined and are 2.29 A (x-axis) and 2.16 A (y-axis). The differences observed have several origins as errors due to the manufacturing of the fixed part, errors on the dimensions and magnetization of the PMs and friction inhomogeneity.

\section{Characterization of the conveyance device}

In Figure 6, a conveyance of the structured plate along $\mathrm{x}$ axis is presented. To realized this test, a $3 \mathrm{~A}$ driving current (higher than the minimum driving current) has been used and a part, used to guide the structured plate during displacement and to avoid straightness error along y-axis, has been fixed on the top side of the DAA. During this experimental test, one controlling sequence has been realized. In Figure 6 (b), the initial plate position and the plate position after the two substeps are shown.

During this test, it has been experimentally observed that the initial plate position has a great influence on the plate displacement. To qualify this influence, a serie of gauge blocks with different dimensions between 14 and $16 \mathrm{~mm}$ have been manufactured. The theoretically perfect initial plate position (as represented in Fig. 4 (a)) is obtained with a $15 \mathrm{~mm}$ gauge block.

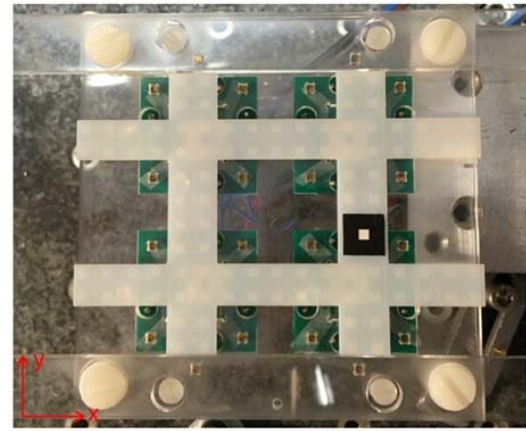

(a)

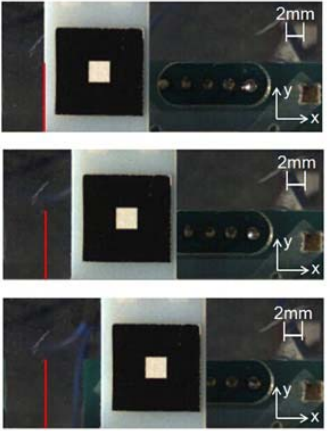

(b)
Fig. 6. Pictures of experimental plate displacement

Using the gauge blocks (Fig. 7), the plate has been placed in different initial positions and its displacement after one displacement sequence has been measured. The results are presented in Figure 8. In this figure, experimental and theoretical results are compared. For the experimental results, the measure has been realized three times for each gauge block dimensions. The errors bars represent the standard deviations calculated from these three measurements. From the figure, it can be easily observed that the gauge block dimension, then the initial plate position, has an influence on the plate displacement. In Figure 4, it can indeed easily been observed that if the structured plate is not well initially positionned, the first displacement plate obtained will be affected because the initial gap between the studs on the MPM and on the structured plate is modified.

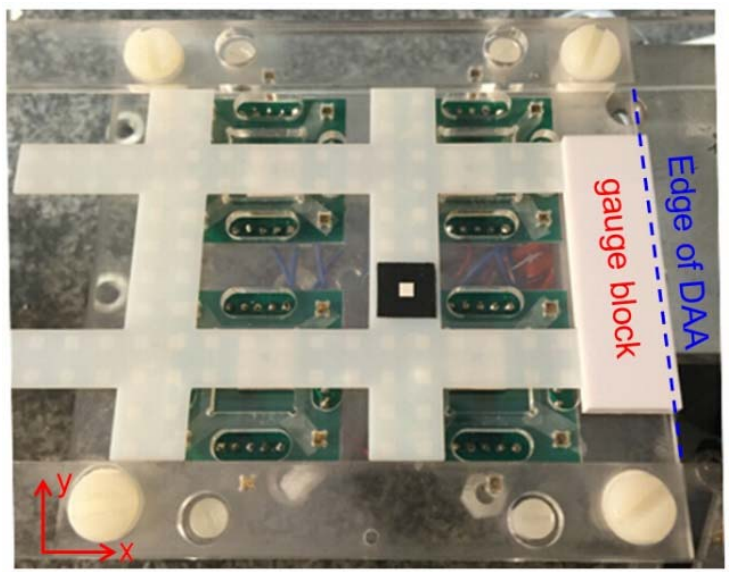

Fig. 7. Prototype with a gauge block for initial positioning of the structured plate

In Figure 9, five controlling sequences have been successively realized in order to move the structured plate in the $+\mathrm{x},-\mathrm{x},-\mathrm{x},-\mathrm{x},+\mathrm{x},+\mathrm{x}$ direction, respectively. For this test, a $3 \mathrm{~A}$ driving current has been used and the $15 \mathrm{~mm}$ gauge block has been used to ensure the initial positioning of the structured plate. The Figure 9 (a) and (b) represent the plate position and displacement as function of the number of controlling sequence, respectively. In this figure, the theoretical and experimental results are ploted. For the different steps, it can be observed that there is a good accordance between the experimental and theoretical results. 


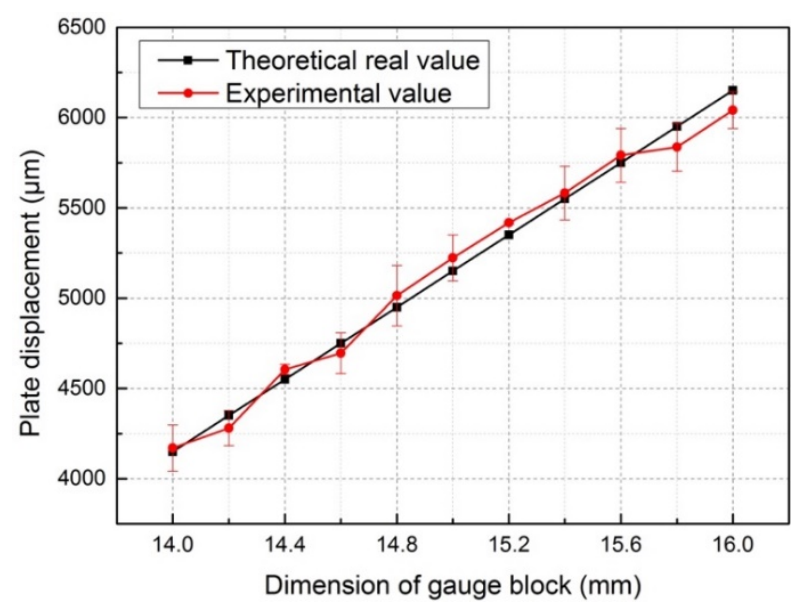

Fig. 8. Influence of the gauge block dimension on the plate displacement

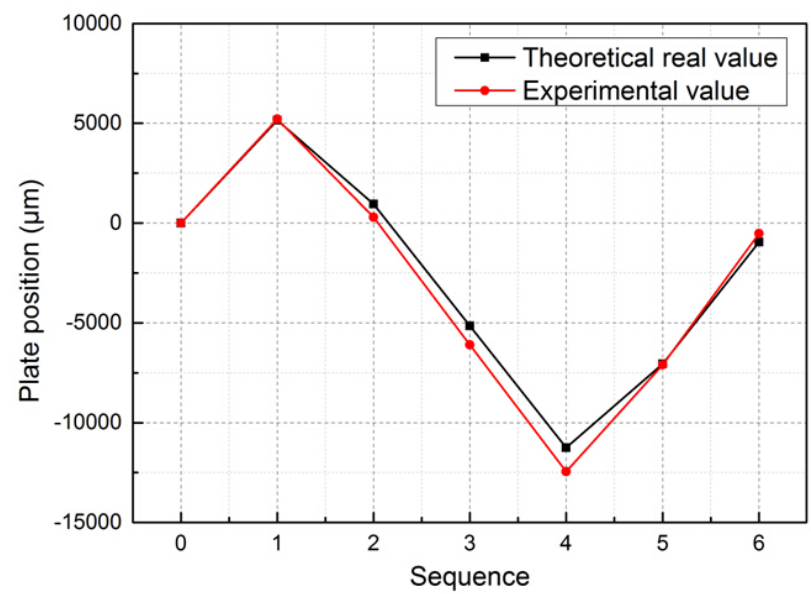

(a)

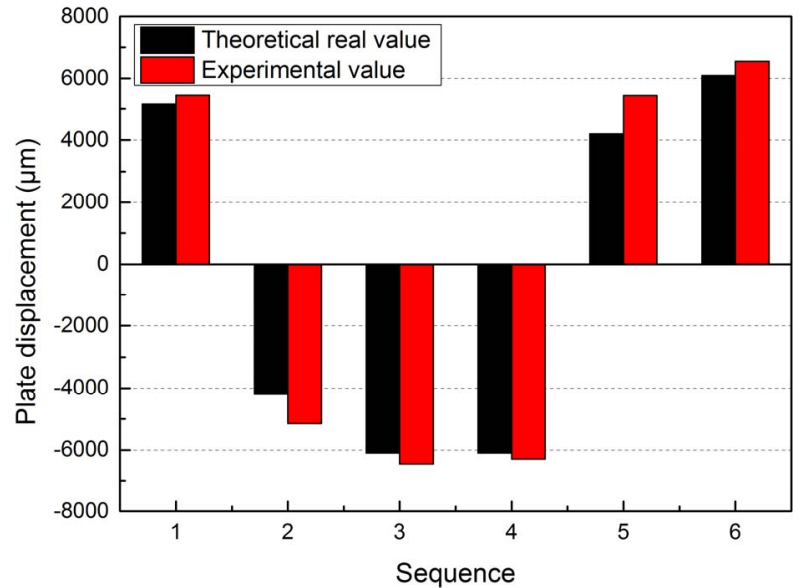

(b)

Fig. 9. Conveyance of the structure plate - a. Plate position for different controlling sequences $-\mathrm{b}$. Plate displacement for different controlling sequences

The influence of the driving current value on the plate displacement has also been studied. For that, the plate displacement after the first sub-step (corresponding to Fig. 4 (c)) is represented with two driving current values: $2.5 \mathrm{~A}$ (Fig. 10 (a)) and 3.5 A (Fig. 10 (b)). During this sub-step, the MPMs
3 and 4 are simultaneously switched in the $+x$ direction to push the structured in this direction. It can be easily observed that with a low driving current value $(2.5 \mathrm{~A})$, the stud on the MPM and the stud on the structured plate are still in contact after the displacement (Fig. 10 (a)). Whereas with a higher driving current value $(3.5 \mathrm{~A})$, the studs are not in contact after the displacement due to a sliding of the structured plate (Fig. 10 (b)). This sliding can be a problem for the engaging step of MPMs 1 and 2 in the second sub-step. In future, a dedicated control will be developed to minimize this sliding while ensuring the MPMs switching and the structured plate displacement.

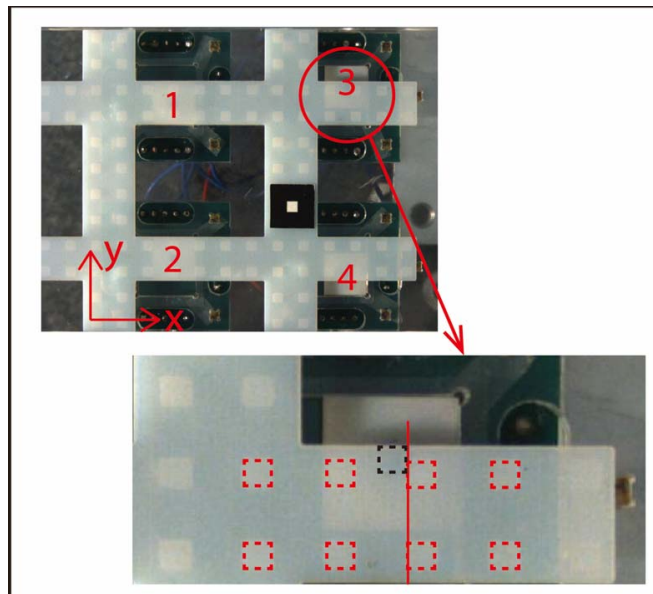

(a)

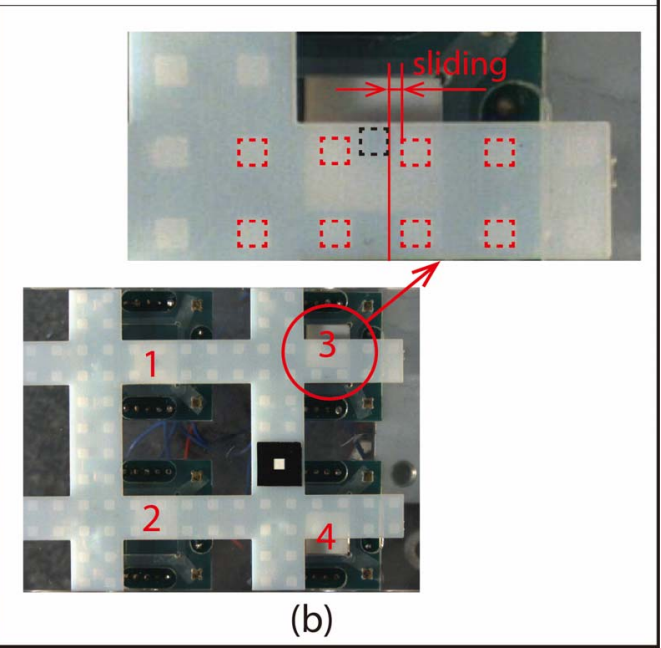

Fig. 10. Influence of the driving current value on the structured plate displacement - a. 2.5 A driving current - b. 3.5A driving current

\section{CONCLUSION AND PERSPECTIVES}

In this paper, a conveyance device based on a DAA and a structured plate has been presented and characterized. The principles of the EDA and of the DAA have been firstly described. The principle of the conveyance device and the control strategy used to generate the conveyance of a structured plate are then presented. Based on the proposed architecture, a prototype has then been manufactured and characterized. The influence of the initial position of the structured plate has been highlighted and quantified and plate displacement along $\mathrm{x}$-axis have been realized in the positive and negative directions. 
Comparisons between theoretical and experimental results have shown a good accordance. The influence of the driving current value on the conveyor behavior has also been tested and a sliding of the structured plate, which can disturb the conveyance task, has been highlighted for high driving current values.

In perspective, a new microfabricated version of this DAA with more EDAs will be realized. The microfabrication technique will be very useful to minimize the manufacturing errors of the prototype. The mechanical part of the device (square cavity) and the structured parts (structured plate and studs) will be microfabricated. With this microfabricated prototype, planar long stroke conveyance tasks of a structured plate will be tested as well as the conveyance of several structured plates on the DAA.

\section{REFERENCES}

[1] H. Ishii, K.L. Ting, "SMA actuated compliant bistable mechanisms," Mechatronics, 2004, pp. 421-437.

[2] Q. Chen, Y. Haddab, P. Lutz, "Mocrofabricated Bistable Module For Digital Microrobotics," Journal of Micro-Nano Mechatronics, vol. 6, 2011, pp. 1-12.

[3] S. Mao, H. Wang, Y. Wu, J. Tang, G. Ding, "A latching bistable microswitch using dual-beam electrothermal actuation," 5th IEEE International Conference on Nano/Micro Engineered and Molecular Systems (NEMS), pp.732-735, 20-23 Jan., 2010.

[4] J. Oberhammer, M. Tang, A.Q. Liu, G. Stemme, "Mechanically tri-stable, true single-pole-double-throw (SPDT) switches", Journal of Micromechanical and Microengineering, vol. 16, pp. 1-8, 2006.

[5] B.T. Chia, C.W. Ma, B.T. Liao, S.C. Shih, Y.J. Yang, "Development of a $2 \times 2$ optical switch using bi-stable solenoid-based actuators," International Conference on Optical MEMS and Nanophotonics (OPT MEMS), pp.123-124, 9-12 Aug., 2010.

[6] Y. Wu, G. Ding, C. Zhang, J. Wang, S. Mao, H. Wang, "Design and implementation of a bistable microcantilever actuator for magnetostatic latching relay", Microelectronics Journal, vol. 6, pp. 325-220, 2010.
[7] P. Cazottes, A. Fernandes, J. Pouget, M. Hafez, "Actuation of bistable buckled beams with Macro-Fiber Composites", IEEE/RSJ International Conference on Intelligent Robots and Systems (IROS), vol. 6, pp. 564-569, 2008.

[8] E.T. Carlen, C.H. Mastrangelo, "Paraffin actuated surface micromachined valves", Thirteenth Annual International Conference on Micro Electro Mechanical Systems (MEMS), pp.381-385, 23-27 Jan., 2000

[9] K.R. Cochran, L. Fan, D.L. DeVoe, "High-power optical microswitch based on direct fiber actuation", Sensors and Actuators A, vol. 119(2), pp. 512-519, 2004

[10] L. Petit, H. Al Hajjar, C. Prelle, F. Lamarque, "Design, modeling and characterization of an optical switch based on four positions digital actuator", IEEE/ASME Transactions on Mechatronics, In Press, 2016.

[11] F. Plötz, S. Michaelis, R. Aigner, H.J. Timme, J. Binder, R. Noé, "A low-voltage torsional actuator for application in RF-microswitches", Sensors and Actuators, A92, pp. 312-317, 2001.

[12] C. Dieppedale, B. Desloges, H. Rostaing, J. Delamare, O. Cugat, J. Meunier-Carus, "Magnetic bistable micro-actuator with integrated permanent magnets", Proceedings of IEEE Sensors, pp. 4, 24-27 Oct., 2004.

[13] C. Jia, J. Zhou, W. Dong, W. Chen, "Design and fabrication of siliconbased $8 \times 8$ MEMS optical switch array", Microelectronics Journal, vol. 40(1), pp. 83-86, 2008

[14] T. Matsunaga, K. Totsu, M. Esashi, Y. Haga, "Tactile display using shape memory alloy micro-coil actuator and magnetic latch mechanism", Displays, vol. 34(2), pp. 89-94, 2013.

[15] S.W. Kim, J.S. Koh, C. Maenghyo, K.J. Cho, "Towards a bio-mimetic flytrap robot based on a snap-through mechanism," IEEE RAS and EMBS International Conference on Biomedical Robotics and Biomechatronics (BioRob), pp.534-539, 26-29 Sept., 2010.

[16] A. Berlin, D. Biegelsen, P. Cheung, M. Fromherz, D. Goldberg, W. Jackson, B. Preas, J. Reich, L.-E. Swartz, "Motion control of planar objects using large-area arrays of MEMS-like distributed manipulators", Micromechatronics'2000, 2000.

[17] L. Petit, A. Hassine, J. Terrien, F. Lamarque, C. Prelle, "Development of a Control Module for a Digital Electromagnetic Actuators Array", IEEE Transactions on Industrial Electronics, vol. 61(9), pp. 4788-4796, 2014.

[18] Jing Xu, L. Petit and C. Prelle, "Design and characterization of a digital actuators array with a structured plate for conveyance application", IEEE International Conference on Mechatronics and Automation (ICMA), pp. 2000-2004, 2015. 